\title{
Modeling and Image Reconstruction in Spectrally Resolved Bioluminescence Tomography
}

\author{
Hamid Dehghani ${ }^{1,2}$, Brian W. Pogue ${ }^{2}$, Scott C. Davis ${ }^{2}$, and Michael S. Patterson ${ }^{3}$ \\ ${ }^{1}$ School of Physics, University of Exeter, UK \\ ${ }^{2}$ Thayer School of Engineering, Dartmouth College, Hanover, NH 03755, USA \\ ${ }^{3}$ Juravinski Cancer Centre and McMaster University, Hamilton, ON, CANADA \\ email:h.dehghani@exeter.ac.uk, Tel:+44 1392 264177,Fax:+44 139226111
}

\begin{abstract}
Recent interest in modeling and reconstruction algorithms for Bioluminescence Tomography (BLT) has increased and led to the general consensus that non-spectrally resolved intensity-based BLT results in a non-unique problem. However, the light emitted from, for example firefly Luciferase, is widely distributed over the band of wavelengths from $500 \mathrm{~nm}$ to $650 \mathrm{~nm}$ and above, with the dominant fraction emitted from tissue being above $550 \mathrm{~nm}$. This paper demonstrates the development of an algorithm used for multi-wavelength $3 \mathrm{D}$ spectrally resolved BLT image reconstruction in a mouse model. It is shown that using a single view data, bioluminescence sources of up to $15 \mathrm{~mm}$ deep can be successfully recovered given correct information about the underlying tissue absorption and scatter.
\end{abstract}

Keywords: Near Infrared Tomography, Bioluminescence Imaging, Image Reconstruction, Finite element Modeling.

\section{INTRODUCTION}

Bioluminescence optical Tomography (BLT) is an emerging imaging technique where the molecular luminescence from Luciferase is used to reconstruct its spatial distribution and to visualize function, physiology or genetic activation within tissue. The light emitted by Luciferase can be detected on the tissue surface using either contact (e.g. optical fibers) or non-contact approaches (e.g. a CCD camera). However, using non-contact methods, the number of available boundary measurements can be significantly larger than those available from using optical fibers. A measured set of emission data forms the basis for tomographic reconstruction of the internal bioluminescence distribution analogous to positron emission tomography (PET) using the mathematics familiar to Near Infrared (NIR) transmission optical tomography. This paper demonstrates the development of an algorithm for modeling of bioluminescence light propagation in tissue and also demonstrates that multi-wavelength or spectrally-resolved bioluminescence tomography (sBLT) is essential for direct depth-resolved recovery of the bioluminescence target distribution.

As a result of the development and widespread implementation of bioluminescence imaging, cancer research has undergone a significant advance in the ability of investigators to determine cell growth in vivo [1]. In vivo bioluminescence imaging involves cells which are tagged with a reporter gene that encodes the sequence for a lightgenerating enzyme, luciferase. Luciferase is produced by the cell, and is a protein that can generate visible light through the oxidation of an enzyme-specific substrate. This process requires the presence of oxygen and ATP as a source of energy. Part of the chemical energy is released as visible light, and this signal becomes an optical indicator of cellular activity in vivo. The signal has inherently low background levels since endogenous light from mammalian cells is essentially zero. Luciferase has, therefore, become a frequently used reporter in many biochemical and cell culture assays. The key is to have a cell line under investigation that has been transfected with the luciferase gene [1]. Studies of tumor metastases have been completed with luciferase imaging, dramatically showing individual events of tumour cell invasion, which could not be observed without this technology [2]. Imaging of wound healing has also exploited the approach to visualize the process over several days [3]. Immune trafficking has been an area of investigation that has lacked appropriate tools to visualize events occurring in vivo. Hardy et al, [4] demonstrated that luciferase bioluminescence from lymphocytes could be used to follow trafficking in vivo. As these tools progress, it is imperative to have improved imaging methods to visualize and quantify the bioluminescence signals in vivo [1,2], in order to gain fundamental insight into tumor growth, regression, immune response and cellular response to therapy. 
Currently, the most advanced techniques only utilize planar surface imaging, which do not provide depth information or the actual activity of the bioluminescence signal. This significant limitation has led to the use of temporal imaging techniques, whereby only the rate of change of the bioluminescence signals over time can be observed. It is essential therefore to improve methods which combine information from disparate imaging technologies to generate improved and increased understanding.

Recent interest in modeling and reconstruction algorithms for BLT has increased [5-7] and led to the general consensus that non-spectrally resolved intensity-based BLT results in a non-unique problem[8]. However, the emitted light from firefly Luciferase is widely distributed over the band of wavelengths from $500 \mathrm{~nm}$ to $650 \mathrm{~nm}$ and above. When not attenuated, it has a peak emission near $560 \mathrm{~nm}$, but when detected from within an animal appears to have a peak closer to $600 \mathrm{~nm}$ with measurable emissions as much as $50 \mathrm{~nm}$ above and below this peak [1]. Using current technology it would be possible to measure the emission at the surface of the tissue in discrete steps of $10 \mathrm{~nm}$ ranging from $550 \mathrm{~nm}$ to $650 \mathrm{~nm}$, although for deeper sources strong optical absorption of tissue at the lower wavelengths might preclude accurate measurements with adequate signal to noise.

This paper outlines the development of a numerical model for SBLT as well as a linear image reconstruction algorithm. Through simulation studies, it is demonstrated that using only a single view data, that is data collected from only one surface side of the model, it is possible to determine the location of bioluminescence signals up to depths of $15 \mathrm{~mm}$. The importance of the underlying tissue absorption and scatter in image reconstruction are also demonstrated, showing that without known or reconstructed values, it is not possible to accurately determine bioluminescence sources.

\section{FORWARD MODEL}

The mathematical basis for sBLT is the Boltzmann transport equation, a physical model for light propagation in tissue. Under the assumption that scattering dominates absorption for red light in tissue, the Boltzmann transport equation can be simplified to the diffusion approximation, which for a continuous light source is given by:

$-\nabla \cdot \kappa(r) \nabla \Phi(r)+\mu_{a}(r) \Phi(r)=B(r)$

where $B(r)$ is an internal bioluminescence source, $\Phi(r)$ is the photon fluence rate at position $\boldsymbol{r}$, and

$\kappa=\frac{1}{3\left(\mu_{a}+\mu_{s}{ }^{\prime}\right)}$

is the diffusion coefficient, $\mu_{a}$ is the optical absorption coefficient and $\mu_{s}{ }^{\prime}$ is the reduced scatter coefficient. A Robin (Type III or mixed) boundary condition is used to account for reflection and refraction at the tissue surface:

$\Phi(\xi, \omega)+2 A \hat{\mathbf{n}} \cdot \kappa(\xi) \nabla \Phi(\xi, \omega)=0$

where $\xi$ is a point on the external boundary, and $A$ depends upon the relative refractive index mismatch between tissue and air. $A$ can be derived from Fresnel's law:

$A=\frac{2 /\left(1-R_{0}\right)-1+\left|\cos \theta_{c}\right|^{3}}{1-\left|\cos \theta_{c}\right|^{2}}$

where $\theta_{c}=\arcsin \left(n_{A I R} / n_{1}\right)$, the angle at which total internal reflection occurs for photons moving from region $\Omega$ with a refractive index $n_{1}$ to air with refractive index $n_{\mathrm{AIR}}$, and

$R_{0}=\frac{\left(n_{1} / n_{A I R}-1\right)^{2}}{\left(n_{1} / n_{A I R}+1\right)^{2}}$ 
At the external boundaries, $n_{A I R}=1$, the refractive index of free space and the refractive index of tissue is assumed to be $\mathrm{n}_{1}=1.33$.

\subsection{FINITE ELEMENT IMPLEMENTATION}

The finite element discretization of a domain $\Omega$ can be obtained by subdividing the domain into $\mathrm{D}$ elements joined at $\mathrm{V}$ vertex nodes. In finite element formalism, $\Phi(\mathrm{r})$ is approximated by the piecewise continuous polynomial function:

$\Phi^{h}(r, w)=\sum_{i}^{V} \Phi_{i} u_{i}(r) \Omega^{h}$

where $\Omega^{h}$ is a finite dimensional subspace spanned by basis functions $\left\{u_{i}(r) ; i=1 \ldots V\right\}$ chosen to have limited support. The problem of solving for $\Phi^{\mathrm{h}}$ becomes one of sparse matrix inversion: in this work, we use bi-conjugate gradients stabilized solver. As developed previously [9, 10], the diffusion equation in the FEM framework can be expressed as a system of linear algebraic equations:

$\left(K(\kappa)+C\left(\mu_{a}\right)+\frac{1}{2 A} F\right) \Phi=B$

where the matrices $\mathrm{K}(\kappa), \mathrm{C}(\mu \mathrm{a})$ and $\mathrm{F}$ have entries given by:

$$
\begin{aligned}
K_{i j} & =\int_{\Omega} \kappa(r) \nabla u_{i}(r) \cdot \nabla u_{j}(r) d^{n} r \\
C_{i j} & =\int_{\Omega} \mu_{a}(r) u_{i}(r) u_{j}(r) d^{n} r \\
F_{i j} & =\oint_{\partial \Omega} u_{i}(r) u_{j}(r) d^{n-1} r
\end{aligned}
$$

and the source vector B has terms

$$
B_{i}=\int_{\Omega} u_{i}(r) B(r) d^{n} r
$$

\subsection{LINEARITY}

An advantage of bioluminescence forward model is that the fluence rate data $\Phi(r)$ can be represented by an operator, which is linear in terms of the bioluminescence source. As an example consider the problem shown in Figure 1. The models on the top row show the internal bioluminescence source distribution for different $2 \mathrm{D}$ models and the bottom row displays the corresponding internal fluence rate. From this example, it is seen that the fluence rate as a function of the combination of the source distribution in the first three 2D models can be achieved by either solving the fluence for the new source distribution or as the summation of the fluence of the individual problems.

\section{IMAGE RECONSTRUCTION}

Given a set of data represented by an operator, which is linear in terms of the bioluminescence source, but nonlinear in terms of optical properties, $y=F\left[\mu_{a}, \kappa, B\right]$, the image reconstruction method is posed as a solution to the following minimization expression:

$\chi^{2}=\arg \min _{\mu_{a}, \kappa}\left\|\left(y-F\left(\mu_{a}, \kappa, B\right)\right)\right\|$

or assuming that absorption and scatter are known, this can be simplified to: 
$\chi^{2}=\arg \min _{\mu_{a}, \kappa}\|(y-F(B))\|$

where $\|$.$\| is the weighted L2-norm, representing the square root of the sum of the squared elements. If the location of$ the bioluminescence source $\mathrm{B}(\mathbf{r})$ is known a priori, one can constrain the problem to calculate the strength of $\mathrm{B}(\mathbf{r})$ to minimize the projection error $\chi^{2}$. However, assuming that no a priori information about $\mathrm{B}(\mathbf{r})$ is known, the problem becomes inherently more difficult. The problem in developing a source location and strength reconstruction algorithm is caused by the fact that the problem statement, equation (1), is linear in terms of its right hand side, $\mathrm{B}(\mathbf{r})$ for both amplitude and position. However, a practical approach to solving this problem is generally developed by assuming that

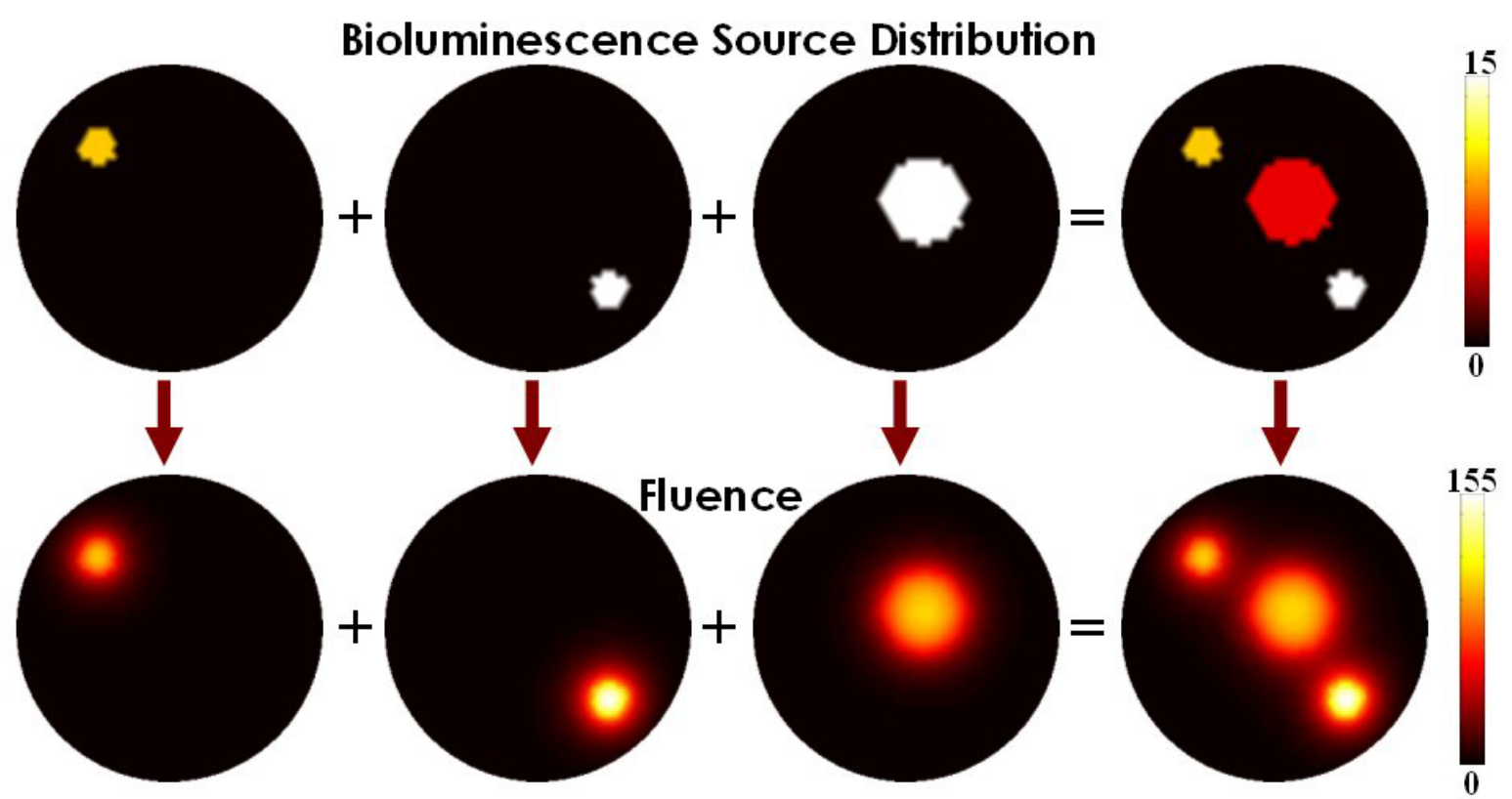

Figure 1. Inherent linearity in Bioluminescence forward problem. The top row shows the internal bioluminescence source distribution for different models and the bottom row displays the corresponding internal fluence rate (logarithm).

the solution to equation 3 can be computed either as a combined solution of all sources, or as the superposition of the two or more sources. These two methods in theory will provide identical solutions. We can take advantage of the linearity of the model by creating a set of independent basis solutions for the source:

$\mathrm{B}=\sum_{i=1}^{N} a_{i} b_{i}$

where the coefficients $a_{i}$ are the weight functions for multiple sources $b_{i}$ at all nodes $i$ in the model containing a total number of nodes $N$. Equation 14 can be represented in matrix form as:

$\mathrm{B}=\mathrm{ab}$

where $\mathbf{b}$ is a matrix of size $\mathrm{N} x \mathrm{~N}$ and a is a vector of length $\mathrm{N}$. Here, each column of matrix $\boldsymbol{b}$ is a unit source point at each appropriate location, and each element of $\boldsymbol{a}$ represents the strength (or weight) of that source. The size of $\boldsymbol{b}$ can be dramatically reduced, if a coarser basis is used for the source than that of the FEM model.

By substituting equation 15 into equation 13 (or 12, although the simple case of known absorption and scatter is discussed for simplicity), and solving for $\boldsymbol{a}$, in a least square manner, we have: 


$$
\left.\mathrm{a}=\mathrm{W}^{\mathrm{T}}(\mathrm{WW})^{\mathrm{T}}\right)^{-1} \mathrm{y}
$$

where $\mathbf{W}$ is a matrix containing the solution of equation 3 for all possible source positions $\mathrm{N}$ and $\mathrm{y}$ is the measured surface boundary data.

Here, $\lambda$ is a regularization parameter and $I$ is the identity matrix. Although the Hessian matrix $W W^{T}$ is invertible, the use of $\lambda$ becomes necessary in the presence of noise in the data. In this work, $\lambda=0.001 \%$ of the maximum of the diagonal of the Hessian is used. Instead of using data from a single wavelength, multi-wavelength sBLT combines datasets that are measured from the same domain containing the same bioluminescence distribution, over a range of usable wavelengths such that

$$
a=\hat{W}^{\mathrm{T}}\left(\hat{\mathrm{W}} \hat{\mathrm{W}}^{\mathrm{T}}\right)^{-1} \hat{\mathrm{y}}
$$

where

$$
\hat{W}=\left[W_{\lambda 1} ; W_{\lambda 2} ; W_{\lambda 3} ; \cdots ; W_{\lambda n} ;\right]
$$

are the weight matrices of all $n$ of cascaded wavelengths and

$\hat{y}=\left[y_{\lambda 1} ; y_{\lambda 2} ; y_{\lambda 3} ; \cdots ; y_{\lambda n} ;\right]$

is the corresponding measured surface data for each wavelength. The solution $a$ is a vector corresponding to the number of unknowns that define the bioluminescence source distribution.

\section{METHODS}

A 3D model of the MOBY mouse [11], Figure 2, was used to generate Bioluminescence data for sources placed deep within the model using the model described above. The 3D FEM model contained 13031 nodes corresponding to 65958 linear tetrahedral elements and a total of 400 detector positions were uniformly distributed in a grid (1 $\mathrm{mm}$ spacing) in the area indicated in Figure 3.
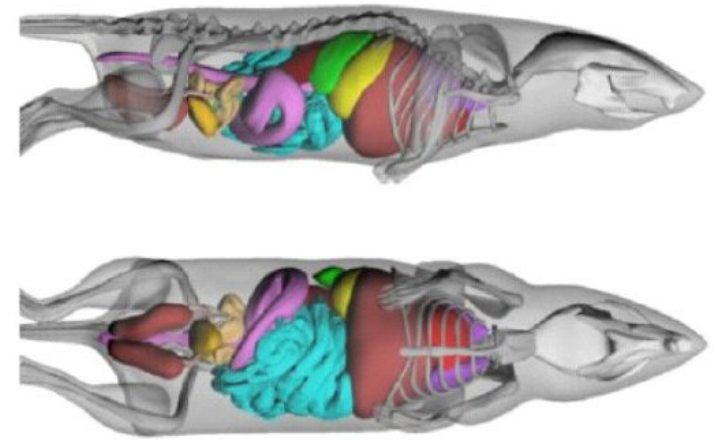

Figure 2. MOBY mouse (Segars et al, Molecular Imaging and Biology, 2004)

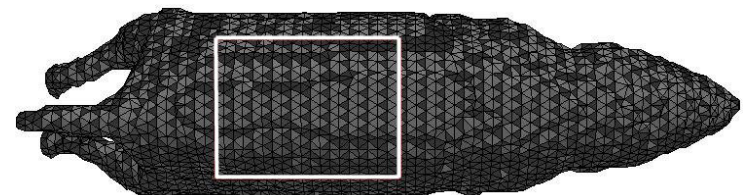

Figure 3. Model of the MOBY mouse used. The white rectangular area indicated the surface area from which boundary data where measured. 
The mouse model for all generated data was assumed to be a heterogeneous model, containing 7 regions. Table 1 shows the assumed regions for the model, together with chromophore concentrations and scattering properties used.

Table 1. The seven regions of anatomy used within the mouse model, together with hromophore concentrations and scattering properties used.

\begin{tabular}{|l|c|c|c|c|c|}
\hline & $\begin{array}{c}\text { Total } \\
\text { hemoglobin } \\
(\mathbf{m M})\end{array}$ & $\begin{array}{c}\text { Oxygen } \\
\text { Saturation } \\
\mathbf{( \% )}\end{array}$ & $\begin{array}{c}\text { Water } \\
\text { concentration } \\
\mathbf{( \% )}\end{array}$ & $\begin{array}{c}\text { Scatter } \\
\text { amplitude }\end{array}$ & $\begin{array}{c}\text { Scatter } \\
\text { power }\end{array}$ \\
\hline Adipose & 0.0033 & 70 & 50 & 0.98 & 0.53 \\
\hline Bones & 0.049 & 80 & 15 & 1.4 & 1.47 \\
\hline Muscles & 0.07 & 80 & 50 & 0.14 & 2.82 \\
\hline Spleen / Liver & 0.3 & 75 & 70 & 0.45 & 1.05 \\
\hline Stomach & 0.01 & 70 & 80 & 0.97 & 0.97 \\
\hline Lungs & 0.15 & 85 & 85 & 1.7 & 0.53 \\
\hline Kidneys & 0.056 & 75 & 80 & 1.23 & 1.51 \\
\hline
\end{tabular}

A number of single bioluminescence sources were placed within the heterogeneous mouse model. In each case, the sources were placed at either 15, 12.5, 10 or $7.5 \mathrm{~mm}$ depths from the top surface of the model (as shown in Figure 5) with a radius of $5 \mathrm{~mm}$. Fluence due to the bioluminescence source was calculated for each individual case, Figure 4 , at a total of 6 wavelengths, ranging from $600-650 \mathrm{~nm}$ in steps of $10 \mathrm{~nm}$.

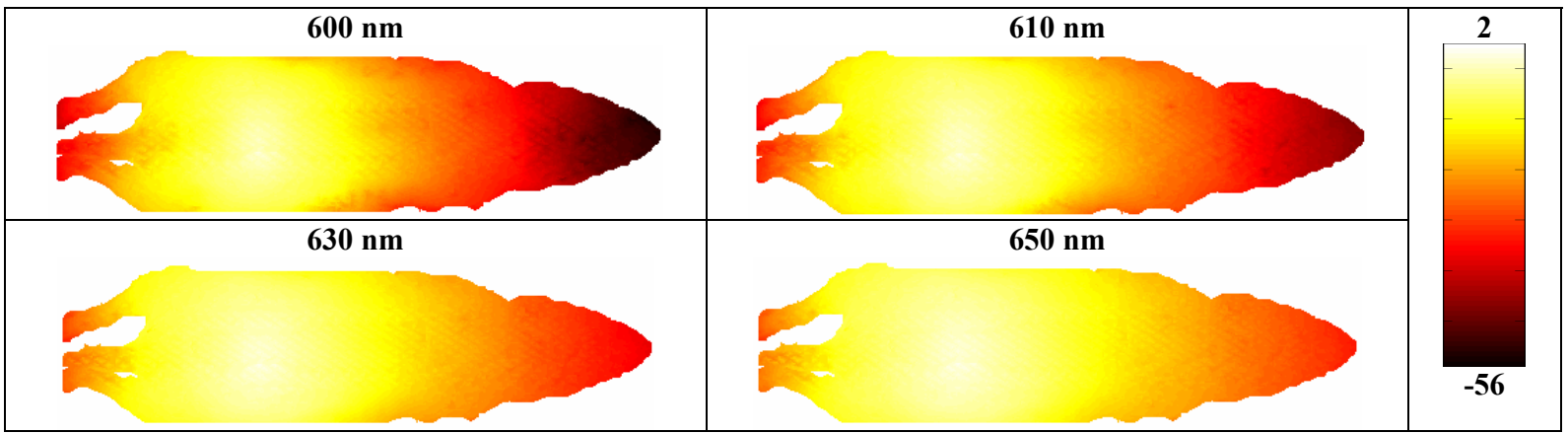

Figure 4. The log of surface fluence due to a single internal source of $5 \mathrm{~mm}$ radius placed at $12.5 \mathrm{~mm}$ beneath the external top surface.

For image reconstruction, boundary data (amplitude) were extracted from the calculated fluence, at the top most surface of the model, as indicated in Figure 3. Images of the bioluminescence source distribution were reconstructed for each case, Figure 5 and 6, using the methods described above, using data from a total of 6 wavelengths, ranging from 600$650 \mathrm{~nm}$ in steps of $10 \mathrm{~nm} .1 \%$ randomly distributed noise was added to the amplitude data. Two different models were considered for image reconstruction:

1. Assuming correct knowledge about the anatomy and optical properties

2. Assuming a homogeneous mouse model which consisted only of adipose tissue.

The time for the calculation of the weight matrix for the model was approximately 4 minutes per wavelength (using a dual Xeon 3.4GHz, 4GB RAM), giving a total reconstruction time of approximately 21 minutes including matrix inversion. 


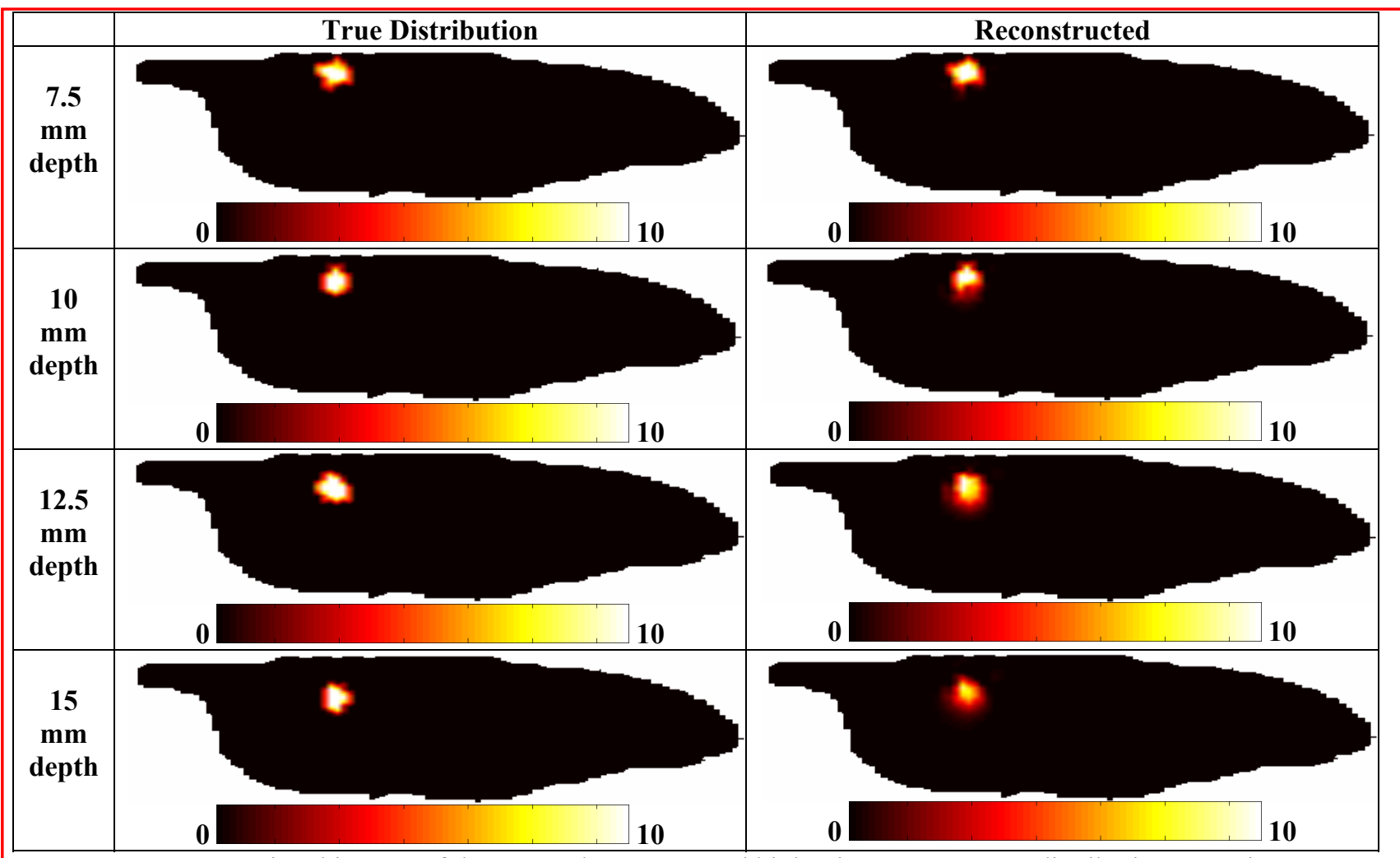

Figure 5. Cross sectional images of the true and reconstructed bioluminescence source distribution assuming correct a-priori information about the anatomy of the model.

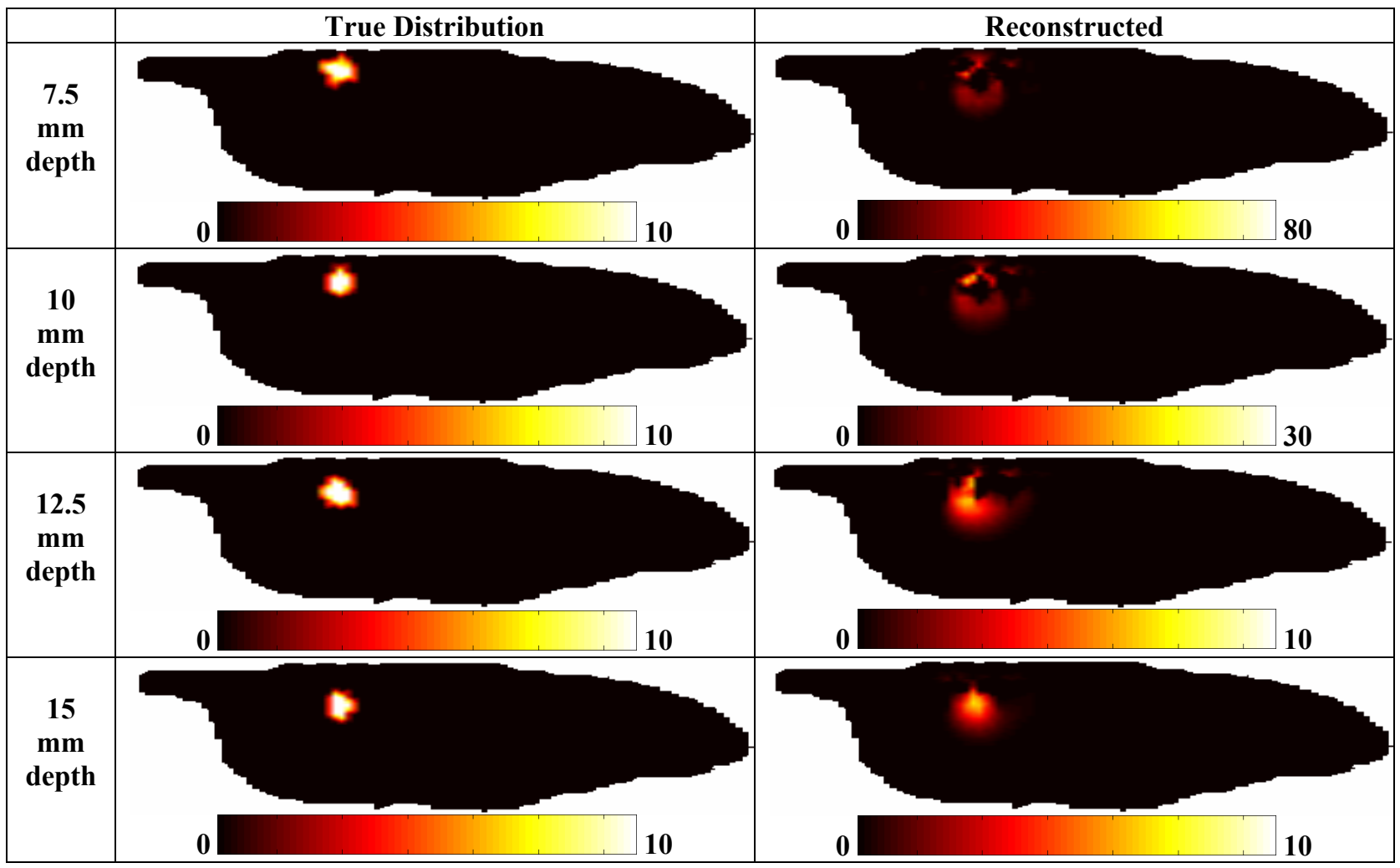

Figure 6. As Figure 5, but assuming a homogeneous model consisting of only adipose tissue.

6434-31 V. 6 (p.7 of 9) / Color: No / Format: A4 / Date: 1/16/2007 6:25:03 AM

SPIE USE:

DB Check,

Prod Check, Notes: 
In order to assess resolution and multiple source separation, 2 sources $(2.5 \mathrm{~mm}$ radius $)$ were modeled at $12.5 \mathrm{~mm}$ depth from the top surface. The distance of separation of the two sources were varied from $20 \mathrm{~mm}$, to $15 \mathrm{~mm}$ and $10 \mathrm{~mm}$. Fluence due to the bioluminescence sources was calculated for each individual case at a total of 6 wavelengths, ranging from $600-650 \mathrm{~nm}$ in steps of $10 \mathrm{~nm}$. For image reconstruction, boundary data (amplitude) were extracted from the calculated fluence, at the top most surface of the model, as indicated in Figure 3. Images of the bioluminescence source distribution were reconstructed for each case, Figure 7 and 8, using the methods described above, with a total of 6 wavelengths, ranging from $600-650 \mathrm{~nm}$ in steps of $10 \mathrm{~nm} .1 \%$ randomly distributed noise was added to the amplitude data. As before two different models were considered for image reconstruction, namely assuming correct knowledge about the anatomy and optical properties or assuming a homogeneous mouse model which consisted of only adipose tissue.

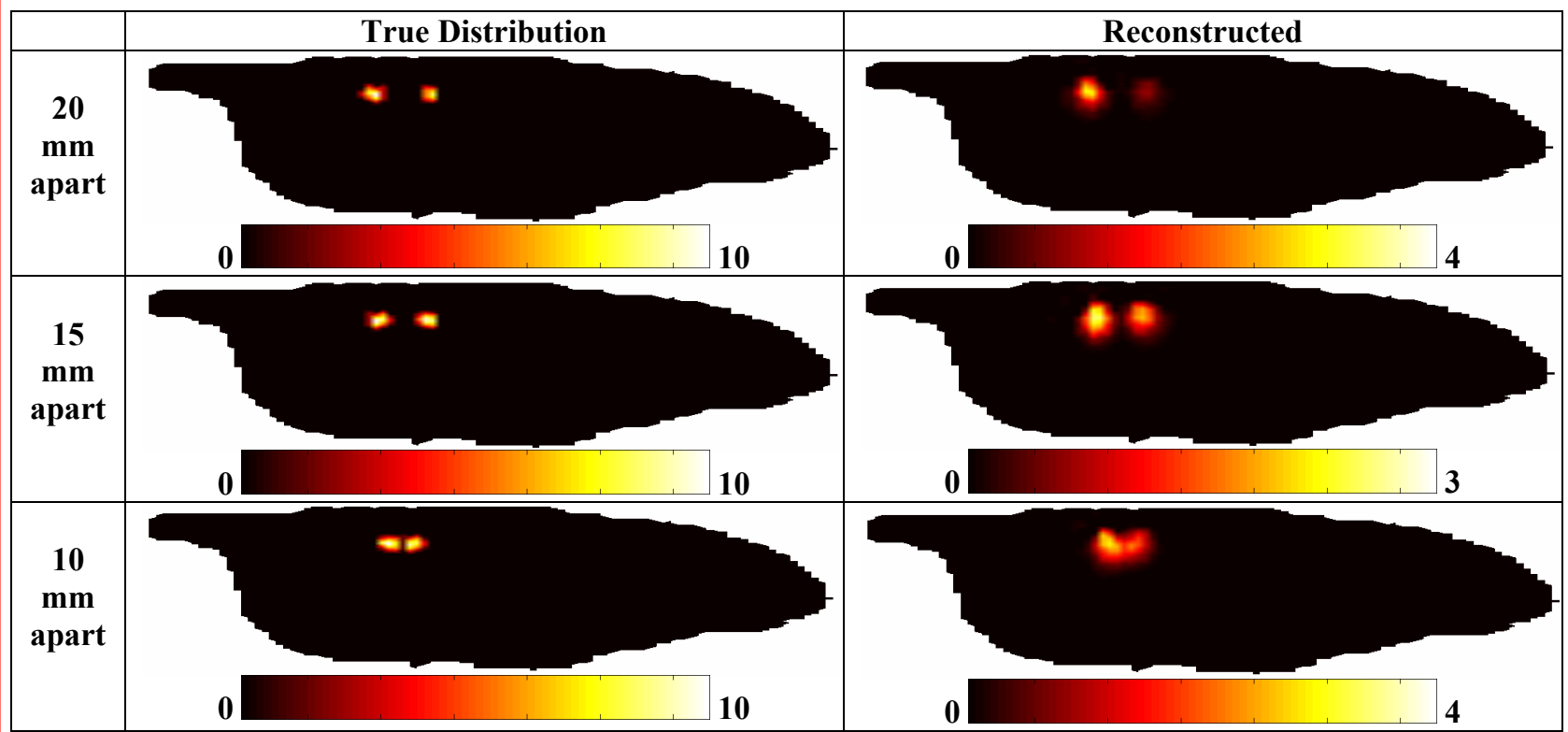

Figure 7. Cross sectional images of the true and reconstructed bioluminescence source distribution assuming correct a-priori information about the anatomy of the model.

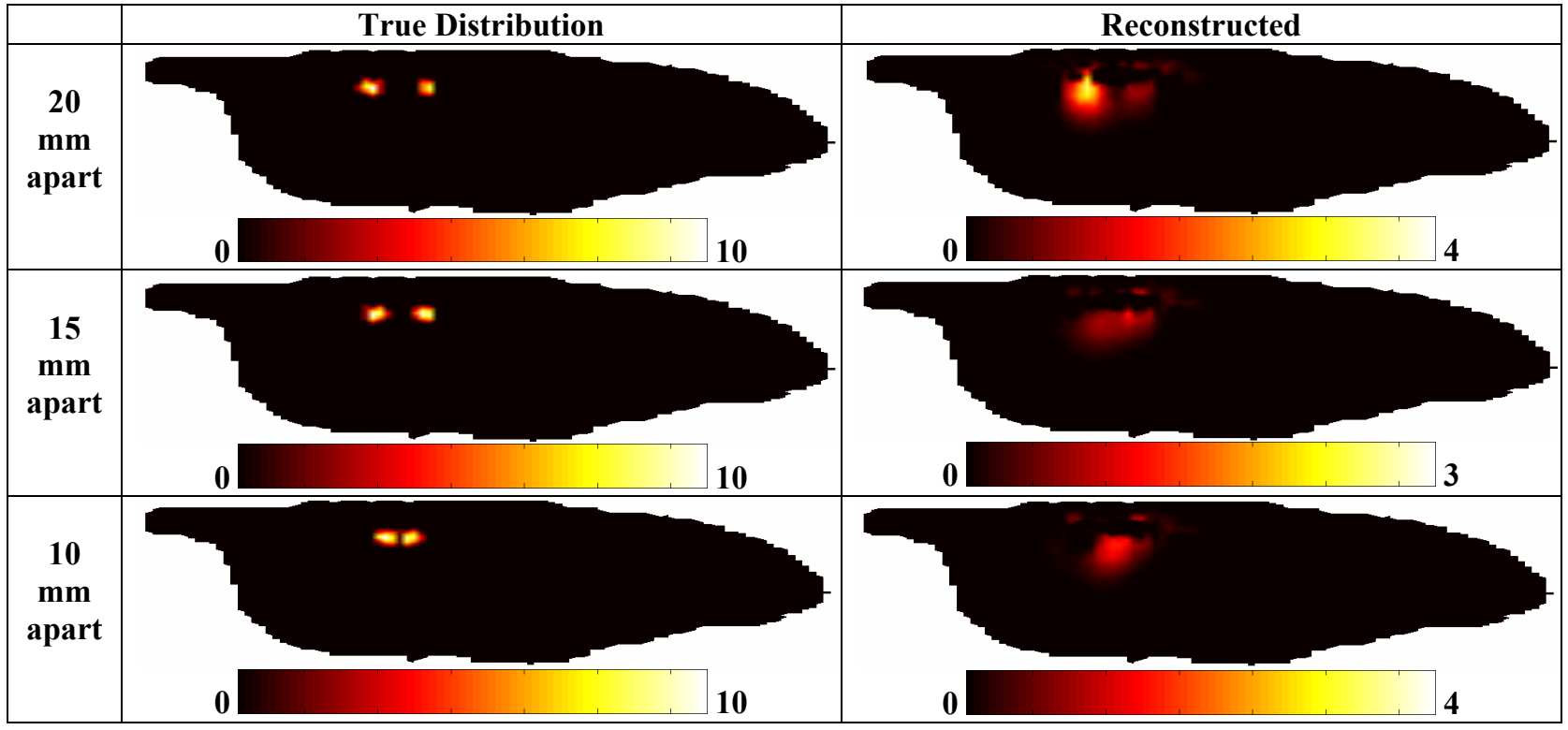

Figure 8. As Figure 7, but assuming a homogeneous model consisting of only adipose tissue. 


\section{DISCUSSIONS AND CONCLUSIONS}

In this paper a modeling and linear single step image reconstruction algorithm for sBLT is presented which demonstrates BLT image recovery from 3D multi-wavelength data. Multi-wavelength emission provides a means of estimating the depth of an object, due to the wavelength dependent attenuation of tissue. Images generated from noisy simulated data have been presented which show that the position of the unknown bioluminescence sources can be reconstructed accurately using a fast linear reconstruction method.

It has been shown that bioluminescence sources can only be accurately quantified in a tomographic geometry when the attenuation due to light propagation is incorporated through either a-priori or absorption-based images of the tissue. In the case of knowing the correct spectral absorption and scattering properties of tissue being imaged, we have shown that bioluminescence sources which are up to $15 \mathrm{~mm}$ deep within a small animal model can be accurately recovered using single surface view data. Without the knowledge of tissue absorption and scattering properties, although an image of bioluminescence sources was recovered, their location and distribution were highly inaccurate. It has also been demonstrated that using a single surface view data, 2 small bioluminescence sources $(2.5 \mathrm{~mm}$ radius $)$ with $10 \mathrm{~mm}$ separation, can be accurately resolved.

\section{ACKNOWLEDGEMENTS}

This work has been sponsored by the National Cancer Institute through grants RO1CA109558 and PO1CA80139, NSERC and the Ontario Cancer Research Network.

\section{REFERENCES:}

1. Contag, C.H., Bachmann, M. H., Adavnces in in vivo bioluminescence imaging of gene expression. Annual Review of Biomedical Engineering, 2002. 4: p. 235-260.

2. Contag, C.H., Ross, B. D., It's not just about anatomy: In vivo bioluminescence imaging as an eyepiece into biology. J Magn Reson Imaging, 2002. 16: p. 378-387.

3. Hamblin, M.R., D. A. O'Donnell, N. Murthy, C. H. Contag and T. Hasan, Rapid control of wound infections by targeted photodynamic therapy monitored by in vivo bioluminescence imaging. Photochemistry \& Photobiology, 2002. 75(1): p. 51-57.

4. Hardy, J., M. Edinger, M. H. Bachmann, R. S. Negrin, C. G. Fathman and C. H. Contag, Bioluminescence imaging of lymphocyte trafficking in vivo. Experimental Hematology, 2001. 29(12): p. 1353-1360.

5. Dehghani, H., Davis, S. C., Jiang, S., Pogue, B. W., Paulsen, K. D., and Patterson, M. S., Spectrally-Resolved Bioluminescence Optical Tomography. Optics letters, 2006. 31(3): p. 365-367.

6. Alexandrakis, G., Rannou, F. R., and Chatziioannou, A. F, Tomographic bioluminescence imaging by use of a combined optical-PET (OPET) system: a computer simulation feasibility study. Phys. Med. Biol., 2005. 50: p. 4225:4241.

7. $\mathrm{Gu}, \mathrm{X}$., Zhang, Q., Larcom, L., Jiang, H., Three-dimensional bioluminescence tomography with model-based reconstruction. Opt. Exp., 2004. 12(17): p. 3996-4000.

8. $\quad$ Wang, G., Li, Y., Jiang, M., Uniqueness theorems in bioluminescence tomography. Med. Phys., 2004. 31(8): p. 2289-2299.

9. Paulsen, K.D., and Jiang H., Spatially varying optical property reconstruction using a finite element diffusion equation approximation. Med. Phys., 1995. 22(6): p. 691-701.

10. Arridge, S.R., M. Schweiger, M. Hiraoka and D. T. Delpy, A Finite Element Approach for Modeling Photon Transport in Tissue. Med. Phys., 1993. 20: p. 299-309.

11. Segars WP, T.B., Frey EC, Johnson GA, and Berr SS, Development of a $4 D$ digital mouse phantom for molecular imaging research. molecular Imaging \& Biology, 2004. 6(3): p. 149-159. 